\title{
Residual inhibition effects with hypersound: a case study
}

\author{
Keywords: residual inhibition, case study, hypersound audio \\ system
}

\section{Case report}

More than 21 million Americans, about $10 \%$ of the entire U.S population, experienced tinnitus in the past year. Of this large group of individuals, it is believed that 1 in 5 suffer from bothersome tinnitus requiring audiological intervention. ${ }^{1}$ Once an underlying medical condition has been ruled out, management options include some combination of counseling and a masking device (sound therapy). These options help manage the debilitating effects with the goal of reducing the patient's reaction to their bothersome tinnitus. ${ }^{2}$

Although most tinnitus management programs are designed to help a patient cope with their negative reactions to tinnitus, the sound therapy component of these programs are intended to provide relief only when the device is on. In the literature, however, there have been reported cases of patients experiencing temporary relief-in rare instances relief lasting several hours-after the masking noise has been turned off. ${ }^{3}$

This post-masking phenomenon, experienced by some individuals with tinnitus, is called residual inhibition. ${ }^{3}$ Residual inhibition (RI) is described as a temporary reduction in loudness of tinnitus as a result of stimulation from a noise. In his classic studies of tinnitus masking, Feldman ${ }^{4}$ observed that a substantial number of individuals suffering from tinnitus experienced a temporary reduction of their tinnitus following the cessation of masking sounds. It is known from previous studies, now more than 20years old, that the duration of RI is typically on the order of tens of seconds, although reports of RI lasting minutes or longer are not uncommon. ${ }^{5}$ In many previous RI studies, masking sounds are presented at levels exceeding the loudness of the patient's tinnitus from 10 seconds to more than 1 minute, and the patient is asked to report the duration of time lapsed before their tinnitus returns.

In many of these RI studies, a majority of patients experience temporary relief from their tinnitus ${ }^{6}$. Meikle and colleagues ${ }^{7}$ tested residual inhibition in 1,451 people found $88 \%$ experienced at least some reduction of their tinnitus after listening to 1 minute of broadband noise at a level moderately $(+10 \mathrm{~dB})$ above the level that just masked their tinnitus. For $57 \%$ of participants, RI was experienced for less than two minutes; and for the remainder, the return of their tinnitus took varying amounts of time-with just 3\% reporting their tinnitus returned in more than 10 minutes. A summary of their findings are shown in Table 1.

Even though it is possible for patients with bothersome tinnitus to experience RI during treatment, it is the general consensus of experts that very few patients actually benefit from this effect. ${ }^{8}$ Some tinnitus experts have speculated that if the masking band of noise could be more effectively shaped by the patient, the duration of residual inhibition could conceivably be transformed into a permanent or semi-permanent condition. ${ }^{9}$

Research to date indicates that RI is a repeatable phenomenon in 80 to $90 \%$ of patients, but in most of these patients their tinnitus
Volume 6 Issue 4 - 2017

\author{
Brian Taylor \\ AT Still University, USA
}

Correspondence: Brian Taylor AT Still University 4I I 3 Beverly Ave, USA, Tel 9523933163, Email brain.taylor.aud@gmail.com

Received: November 07, 2016 | Published: March 27, 2017

returned in less than 2 minutes. There are, however, a small percentage of individuals who experience the effects of RI for several hours or even days. ${ }^{8}$

Table I The reported residual inhibition times after listening to masking noise for I minute ${ }^{7}$

\begin{tabular}{ll}
\hline $\begin{array}{l}\text { Reported Residual } \\
\text { Inhibition }\end{array}$ & $\begin{array}{l}\% \text { Patients } \\
\text { Reporting RI }\end{array}$ \\
\hline Less than 2 Minutes & $57 \%$ \\
2 to 4 Minutes & $23 \%$ \\
4 to 6 Minutes & $11 \%$ \\
6 to 8 Minutes & $4 \%$ \\
8 to I0 Minutes & $2 \%$ \\
More than 10 Minutes & $3 \%$ \\
\hline
\end{tabular}

The purpose of this case study was to investigate RI in a group of individuals with bothersome tinnitus, using a recently launched tinnitus masking device. The Hypersound Audio System (HSS) functions by creating a beam of sufficiently high-intensity ultrasound which demodulates in the air column to form a beam of audible sound. Given the way Hypersound works, no ear level apparatus is worn by patients-they simply sit in the beam to use it. Hyper Sound, first released in 2015 as a customizable directed audio device, primarily used for at-home television viewing, employs ultrasonic, proprietary processing techniques. ${ }^{10}$ Recently, the Hypersound Audio System added nine noises that can be adjusted by the end-user for the purposes of masking tinnitus. Recently, the tinnitus masking feature on-board Hyper Sound received FDA clearance and a study demonstrated its ability to mask tinnitus during stimulus. ${ }^{11}$

\section{Methods}

In order to study the effects of Hypersound on residual inhibition, 18 individuals were randomly selected from a pool of patients in a private practice audiology clinic in Saskatchewan, Canada. All 18 patients had completed a basic audiometric evaluation and received at least one prior consultation with an audiologist about their tinnitus.

To be included in the study, patients had to meet the following criteria

a. At least 18 years of age. 
b. Mild to moderately severe high frequency sensorineural hearing loss.

c. Commitment to completing the 4 appointment study

d. Completion of hearing assessment, outcome questionnaires, and tinnitus match for frequency, loudness and minimum masking level.

e. Experiencing tinnitus for a minimum of 3 months.

\section{Exclusion criteria}

a. Not undergoing any other tinnitus treatment/therapy concurrently.

b. Experiencing tinnitus related to a medical disorder that was still under investigation.

c. Reporting pulsatile tinnitus, objective tinnitus, or low frequency tinnitus.

d. Reporting any major or medical psychiatric conditions.

Each study participant was given instructions on how the HyperSound system works. Using the adjustable tinnitus program, each participant adjusted the HyperSound-transmitted stimulus to mask their tinnitus. Based on the frequency match of the patient's tinnitus, the clinician chose a sound source that was comfortable for the participant to listen to and adjusted the frequency response of the amplifier. The subjects then sat and listened to the audible HyperSound beam for 20 minutes. The volume of the masking stimulus was initially set at the start of each subsequent appointment by the clinician. Dependent on RI results, the clinician would change and/or adjust the volume, frequency response and sound source at the next appointment to try to achieve better RI results. Subsequently, this procedure was repeated four times on four separate days on all 18 study participants. Each participant's 20minute session with HyperSound was at least 7 days apart. At the conclusion of each 20 minute exposure, study participants were asked to report the approximate length of their residual inhibition. Each study participant reported the duration of their residual inhibition at their next appointment Table 2 shows the results of RI times for 18 participants for each of their four clinic visits.

Table 2 The self-reported RI effects of all 18 participants following 20 minutes of exposure to HyperSound masking noise

\begin{tabular}{ll}
\hline $\begin{array}{l}\text { Study } \\
\text { Participant } \\
\text { Number }\end{array}$ & Self-Reported Length of Residual Inhibition Effects \\
\hline I & Visit I: 3 -4 hours \\
& Visit 2: $3-4$ hours \\
& Visit 3: $3-4$ hours \\
& Visit $4: 3-4$ hours \\
& Visit I: 12 hours \\
& Visit 2: I2 hours \\
& Visit 3: I2 hours \\
& Visit 4: 12 hours \\
& Visit I: no change \\
& Visit 2: no change \\
& Visit 3: no change \\
& Visit 4: no change \\
& Visit I: $24-36$ hours \\
& Visit 2: $24-36$ hours \\
& Visit 3: $24-36$ hours \\
& Visit 4: $24-36$ hours \\
\hline
\end{tabular}

Table Continued...

\begin{tabular}{|c|c|}
\hline $\begin{array}{l}\text { Study } \\
\text { Participant } \\
\text { Number }\end{array}$ & Self-Reported Length of Residual Inhibition Effects \\
\hline \multirow[t]{4}{*}{5} & Visit I: 20 minutes \\
\hline & Visit 2: 15 minutes \\
\hline & Visit 3: 5 minutes \\
\hline & Visit 4: 5 minutes \\
\hline \multirow[t]{4}{*}{6} & Visit I: no change \\
\hline & Visit 2: no change \\
\hline & Visit 3: no change \\
\hline & Visit 4: no change \\
\hline \multirow[t]{4}{*}{7} & Visit I: 5 minutes \\
\hline & Visit 2: 5 minutes \\
\hline & Visit 3: 5 minutes \\
\hline & Visit 4: 5 minutes \\
\hline \multirow[t]{4}{*}{8} & Visit I: 20 minutes \\
\hline & Visit 2: 20 minutes \\
\hline & Visit 3: 20 minutes \\
\hline & Visit 4: 20 minutes \\
\hline \multirow[t]{4}{*}{9} & Visit I: no change \\
\hline & Visit 2: no change \\
\hline & Visit 3: no change \\
\hline & Visit 4: no change \\
\hline \multirow[t]{4}{*}{10} & Visit I: 5 minutes \\
\hline & Visit 2: 5 minutes \\
\hline & Visit 3: 3 minutes \\
\hline & Visit 4: 10 minutes \\
\hline \multirow[t]{4}{*}{11} & Visit I: no change \\
\hline & Visit 2: no change \\
\hline & Visit 3: no change \\
\hline & Visit 4: no change \\
\hline
\end{tabular}

\section{Results and discussion}

The majority of participants experienced RI with 11 (61\%) participants reporting post masking RI effects. Moreover, four of the participants reported RI effects lasting three or more hours. These findings are consistent with previous investigations $6,7,10$ in which RI effects were reported to be between one minute and several hours. Of the 11 subjects which experienced RI, seven (64\%) reported the length of inhibitory effects was the same following all four 20-minute exposure times, which demonstrates the remarkable consistency of the effect. Unlike Meikle et al., ${ }^{7}$ in which $80 \%$ of participants reported RI effects lasting four minutes or less, results of this case study suggest a higher percentage of individuals with tinnitus may experience RI effects of longer duration after 20minutes of exposure to masking noise with HyperSound.

The longer duration of the post masking effects of some study participants reported here relative to other studies may be result from longer exposure times to the masking noise, as the other studies ${ }^{7,9}$ that examined RI effects used masking noise of shorter duration. Another possible explanation for the longer post masking effects experienced by some of the participants in this case study might be related to the customization of the masking sounds. One report ${ }^{8}$ suggests residual inhibitory effects may increase in individuals that can temporarily mask their tinnitus with broader band signals. Given the extended, broadband frequency response $(>12 \mathrm{KHz}$ ) of HyperSound, it is possible the longer post masking effects experienced by some individuals may benefit from this unique feature. Further investigations are warranted to better understand the relationship between the HyperSound masking noises, their ultra-high frequency characteristics and potential post masking effects. 
This case study suggests some patients may experience temporary relief from their bothersome tinnitus after 20 minutes of exposure to a customizable masking noise transmitted by HyperSound. Given the majority of participants in this case study experienced RI effects lasting several minutes to, in a few cases, over a day, further studies are warranted to better understand the potential residual inhibitory effects of HyperSound for some patients.

\section{Acknowledgments}

None.

\section{Conflicts of interest}

Author declares there are no conflicts of interest.

\section{Funding}

None.

\section{References}

1. Bhatt. JAMA Otolaryngol Head Neck Surg. 2016.

2. Walker DD, Cifu AS, Gluth MB. Tinnitus. JAMA. 2016;315(20):2221-2222.
3. Tyler R. Tinnitus Treatment: Clinical Protocols. Thieme Publishing, New York, USA. 2006. p. 240.

4. Feldmann H. Homolateral and contralateral masking of tinnitus by noise-bands and by pure tones. Audiology. 1971;10(3):138-144.

5. Vernon JA, Meikle MB. Tinnitus Masking: Unresolved Problems. Ciba Found Symp. 1981;85:239-262.

6. Larry E Roberts, Jos J Eggermont, Donald M Caspary, et al. Ringing ears: The neuroscience of tinnitus. Journal of Neuroscience. 2010;30(45):14972-14979.

7. Meikle MB, Creedon TA, Griest SE. Tinnitus Archive, second edition. Oregon Hearing Research Center, Portland, USA. 2004.

8. Henry James A. Measurement of tinnitus. Otol \& Neurolol. 2016;37(8):276-285.

9. Henry JA, Rheinsburg B, Zaugg T. Comparison of custom sounds for achieving tinnitus relief. J Am Acad Audiol. 2004;15(8):585-598.

10. Mehta R, Mattson S, Seitzman R, et al. Speech recognition in the sound field: directed audio vs. conventional speakers. Audiology Online. 2015.

11. Mehta R, Mattson S, Kappus B. Treatment of Tinnitus Using a Customized, Ultrasonic Acoustic Stimulus Delivered via the HyperSound Audio System. Audiology Online. 2016. 